\title{
Radiation reaction in quantum field theory
}

\author{
Atsushi Higuch* \\ Department of Mathematics, University of York, \\ Heslington, York YO10 5DD, United Kingdom
}

(Dated: March 30, 2004)

\begin{abstract}
We investigate radiation-reaction effects for a charged scalar particle accelerated by an external potential realized as a space-dependent mass term in quantum electrodynamics. In particular, we calculate the position shift of the final-state wave packet of the charged particle due to radiation at lowest order in the fine structure constant $\alpha$ and in the small $\hbar$ approximation. We show that it disagrees with the result obtained using the Lorentz-Dirac formula for the radiation-reaction force, and that it agrees with the classical theory if one assumes that the particle loses its energy to radiation at each moment of time according to the Larmor formula in the static frame of the potential. However, the discrepancy is much smaller than the Compton wavelength of the particle. We also point out that the electromagnetic correction to the potential has no classical limit. (Correction. Surface terms were erroneously discarded to arrive at Eq. (59). By correcting this error we find that the position shift according to the Lorentz-Dirac theory obtained from Eq. (12) is reproduced by quantum field theory in the $\hbar \rightarrow 0$ limit. We also find that the small $V(z)$ approximation is unnecessary for this agreement. See Sec. VII)

PACS numbers: 03.65.-w, 12.20.-m
\end{abstract}

\section{INTRODUCTION}

Although classical electrodynamics is a well established theory, there has been some controversy over the nature of the reaction of a point charge to its own radiation. The standard equation for the radiation-reaction force, the Lorentz-Dirac equation 1, 2], admits runaway solutions which describe the charged particle accelerating on its own and reaching the speed of light in a very short time. (See, e.g., Refs. 3, 4] for a review of the LorentzDirac theory.) Although it has been shown that there is a unique solution that does not exhibit the runaway behaviour for any bounded time-dependent force acting only for a finite interval of time [5] (see also Refs. [3, 6] ), this solution violates causality. It is generally believed that these problems will be absent once the finite-size effect is properly taken into account. For example, an analysis of a charged sphere has led Yaghjian [] to the conclusion that the radiation-reaction 4 -force is modified at the onset of acceleration in such a way that there is no causality violation in the unique non-runaway solution. There is also an approach based on "reduction of order" [8] which excludes runaway solutions without introducing causality violation. In this method the radiation-reaction force is re-expressed in terms of the external force. This approach has recently been advocated by several authors 9, 10].

Thus, it may be fair to say that the aspects of the Lorentz-Dirac theory that were traditionally regarded as problematic have been clarified. However, there are other counter-intuitive aspects in the Lorentz-Dirac theory which are not discussed very often. These aspects

*Electronic address: ah28@york.ac.uk persist in reformulations of the Lorentz-Dirac equation mentioned before. For example, the radiation-reaction force vanishes if the acceleration of the charge is constant. Although this is not so serious a problem as existence of runaway solutions or causality violation, it is certainly counter-intuitive since the charge radiates energy away continuously while being accelerated. A related fact is that the radiation-reaction force makes the kinetic energy of the charge increase if the acceleration of the charge increases in time, as we will see in the next section, despite the fact that the charge is radiating in the meantime.

In view of these counter-intuitive features of the Lorentz-Dirac theory, it is natural to ask whether or not this theory reproduces the classical limit for a point charge in quantum electrodynamics (QED). (Moniz and Sharp have studied QED in this context and concluded that the Lorentz-Dirac theory is reproduced in the classical limit by studying the Heisenberg equations for an extended charge and taking the zero-size limit [11].) It is well known that the loopwise expansion in quantum field theory is an expansion in powers of $\hbar[12,13]$. Therefore the classical limit $\hbar \rightarrow 0$ in this sense is the tree approximation. However, one should not use this limit in the problem at hand. For example, this limit is taken with the mass parameter $m c / \hbar$ held fixed (see, e.g., Ref. [14]). Thus, the mass $m$ also tends to zero in this limit. In the radiation-reaction problem the mass as well as the electric charge $e$ must be kept fixed in the limit $\hbar \rightarrow 0$. Therefore, the limit we would need to consider is certainly not the tree approximation and, in fact, does not exist as emphasized recently by Julia [15]. This fact can readily be seen by noting that the fine structure constant $\alpha=e^{2} / 4 \pi \hbar c \approx 1 / 137$ is of order $\hbar^{-1}$. Thus, perturbation theory would break down in the $\hbar \rightarrow 0$ limit with $e$ fixed. For example, the one-loop correction to the electron magnetic moment, $\alpha / 2 \pi$, would diverge despite the 
fact that it is a very small fixed number.

Although the limit $\hbar \rightarrow 0$ with $m$ and $e$ fixed would be divergent, one can still compare some quantities in QED with classical ones at order $\alpha$. At this order in $\alpha$, physical quantities of interest are of finite order in $\hbar^{-1}$, and there are some quantities of order $\hbar^{0}$, which can be compared with the corresponding quantities in classical electrodynamics. We note, however, that these quantities are much smaller in reality than some quantities which vanish in the limit $\hbar \rightarrow 0$. The following example illustrates this paradoxical feature. Let $\omega$ be the frequency of an electromagnetic wave. Then the energy of a single photon $\hbar \omega$ tends to zero as $\hbar \rightarrow 0$, but $\alpha \hbar \omega=e^{2} \omega / 4 \pi c$ remains finite. Thus, the quantity $\alpha \hbar \omega$ is meaningful in classical electrodynamics even though it is much smaller than $\hbar \omega$.

In this paper we examine the behaviour of a wave packet of a charged scalar field undergoing acceleration due to an external potential realized as a position dependent mass term to first order in $\alpha$. In particular, we calculate the change in the position of the final-state wave packet, the position shift, as a result of radiation using the WKB approximation. We demonstrate that the $\hbar \rightarrow 0$ limit of this quantity disagrees with the corresponding result in the Lorentz-Dirac theory. Then we show that it agrees instead with the result in classical electrodynamics obtained by assuming that the kinetic energy of the particle is lost to the radiation given by the classical Larmor formula at each moment of time in the rest frame of the potential. We also point out that the electromagnetic correction to the potential is of order $\hbar^{-1}$ and, therefore, cannot be calculated in classical electrodynamics. The rest of the paper is organized as follows. In Sec. II we review the Lorentz-Dirac theory and calculate the position shift of a charged particle due to radiation in the nonrelativistic approximation in this theory. In Sec. III we calculate the transition amplitude for a charged scalar particle emitting a photon using the WKB approximation. This is used in Sec. IV to calculate the position shift in scalar quantum electrodynamics. Here, we closely follow an unpublished paper [16]. Then in Sec. $\mathbb{\nabla}$ we examine the one-loop effect (with no emission of photons) which results in a correction to the external potential. We summarize our results and make some concluding remarks in Sec. VI] The calculations are performed using conveniently chosen physical polarization vectors for photons, but they are shown to agree with the results obtained without choosing physical polarizations in Appendix A. A formula used to compute the position shift is justified in Appendix B. Our metric signature is +--- .

\section{THE POSITION SHIFT IN THE LORENTZ-DIRAC THEORY}

We first motivate the standard Lorentz-Dirac theory briefly. We do not derive it but describe how it natu- rally arises in classical electrodynamics. (See, e.g. Ref. 4] for a derivation.) Suppose that a classical charged particle with charge $e$ and 4-velocity $u^{\mu}$ is accelerated by an external 4 -force $F_{\text {ext }}^{\mu}$. [The 4 -velocity of a particle moving in the $z$-direction with speed $v$ is $(\gamma c, 0,0, \gamma v)$ with $\gamma \equiv\left(1-v^{2} / c^{2}\right)^{-1 / 2}$. Note that $u^{\mu} u_{\mu}=c^{2}$.] The 4-velocity $u^{\mu}$ satisfies the following equation:

$$
m \frac{d u^{\mu}}{d \tau}=F_{\mathrm{ext}}^{\mu}
$$

where $m$ is the rest mass of the particle and where $\tau$ is the proper time along the world line of the particle. The particle emits electromagnetic radiation with 4-momentum $P^{\mu}=(E / c, \mathbf{P})$, where $E$ is the energy and $\mathbf{P}$ is the momentum, given by the relativistic generalization of the Larmor formula:

$$
\frac{d P^{\mu}}{d \tau}=\frac{e^{2}}{6 \pi c^{5}} a^{2} u^{\mu},
$$

where $a=\sqrt{-\dot{u}^{\mu} \dot{u}_{\mu}}$ (with $\dot{u}^{\mu}=d u^{\mu} / d \tau$ ) is the proper acceleration of the particle (see, e.g., Ref. 3, 4]). Since it is the particle that radiates, one expects that its 4momentum should be reduced by the amount carried away by the radiation. It is natural to describe this effect in terms of a 4 -force. Thus, one is led to modify the equation of motion of the particle as

$$
m \frac{d u^{\mu}}{d \tau}=F_{\mathrm{ext}}^{\mu}+K^{\mu}
$$

where $K^{\mu}$ is the radiation-reaction 4 -force which represents the back reaction of the radiation on the charged particle. It may seem that the 4 -force $K^{\mu}$ should be equal to the negative of the right-hand side of Eq. (2) because of energy-momentum conservation. However, this cannot be the case because this 4-force would not satisfy the condition $u_{\mu} K^{\mu}=0$, which is a consequence of the equation $u_{\mu} \dot{u}^{\mu}=0$. Therefore, one is led to require only that the total loss of 4-momentum of the particle be equal to the total 4-momentum radiated away assuming that the acceleration occurs only for a finite time. Thus, one requires that

$$
K^{\mu}=-\frac{e^{2}}{6 \pi c^{5}} a^{2} u^{\mu}+\frac{d C^{\mu}}{d \tau}
$$

for some vector $C^{\mu}$ because the second term does not contribute to the total change in the 4-momentum, $\int_{-\infty}^{+\infty} K^{\mu} d \tau$. By using the condition $u_{\mu} K^{\mu}=0$ one arrives at the following equation:

$$
u_{\mu} \frac{d C^{\mu}}{d \tau}=\frac{e^{2}}{6 \pi c^{3}} a^{2} .
$$

Noting that $a^{2}=-\dot{u}_{\mu} \dot{u}^{\mu}=u_{\mu} \ddot{u}^{\mu}$, one finds

$$
\frac{d C^{\mu}}{d \tau}=\frac{e^{2}}{6 \pi c^{3}} \frac{d^{2} u^{\mu}}{d \tau^{2}}+\frac{d \tilde{C}^{\mu}}{d \tau}
$$


where $d \tilde{C}^{\mu} / d \tau$ is orthogonal to $u^{\mu}$. Letting $\tilde{C}^{\mu}=0$, one arrives at

$$
K^{\mu}=\frac{e^{2}}{6 \pi c^{3}}\left(\frac{d^{2} u^{\mu}}{d \tau^{2}}-\frac{1}{c^{2}} a^{2} u^{\mu}\right) .
$$

This is known as the Abraham 4-vector [17], and Eq. (3) with this 4 -force is the Lorentz-Dirac equation.

To illustrate counter-intuitive features of this equation let us consider one-dimensional motion parametrized by the rapidity $\beta$ with $u^{\mu}=\left(u^{0}, u^{1}\right)=(c \cosh \beta, c \sinh \beta)$ and $F_{\text {ext }}^{\mu}=\left(\tilde{F}_{\text {ext }} \sinh \beta, \tilde{F}_{\text {ext }} \cosh \beta\right)$. Then Eq. (3) reads

$$
\frac{d \beta}{d \tau}=\frac{1}{m c} \tilde{F}_{\text {ext }}+\frac{e^{2}}{6 \pi m c^{3}} \frac{d^{2} \beta}{d \tau^{2}} .
$$

The second term represents the radiation-reaction force. Notice that it vanishes if the acceleration is constant and is positive if the acceleration increases. Thus, the radiation-reaction force pushes the particle forward if the acceleration increases even though the particle is radiating. This is rather counter-intuitive.

Now, let us describe the classical counterpart of our model. In this model a particle with mass $m$ and charge $e$ moves in the $z$-direction from $z=-\infty$ to $z=+\infty$ and is accelerated by a static potential $V(z)$ whose derivative is nonzero only in a finite interval. We define $V_{-\infty} \equiv V(-\infty)$ and let $V(+\infty)=0$. We work in the nonrelativistic approximation. Thus, the energy is given by $\frac{1}{2} m v^{2}+V(z)$, where $v$ is the velocity of the particle. We assume that the kinetic energy is much larger than the potential energy, and we retain only the terms up to second order in $V(z)$. Note that the particle moves in the positive $z$-direction forever under this assumption. We assume also that at $t=0$ the particle has passed the region with $V^{\prime}(z) \neq 0$ and that it moves at constant velocity for $t>0$. Let us denote the position and velocity of the particle without radiation by $\tilde{z}$ and $\tilde{v}$, respectively, and define $\delta z \equiv z-\tilde{z}$ and $\delta v \equiv v-\tilde{v}$. One has $\left.\delta z\right|_{t=-\infty}=0$ and $\left.\delta v\right|_{t=-\infty}=0$ by definition. We will calculate the position shift due to radiation at $t=0$ denoted by $\left.\delta z\right|_{t=0}$ using the Lorentz-Dirac equation. We emphasize that this position shift is measured after the acceleration because there is no acceleration for $t>0$ by assumption.

The rate of change in the energy of the particle according to the Lorentz-Dirac theory is

$$
\frac{d}{d t}\left[\frac{1}{2} m v^{2}+V(z)\right]=\frac{e^{2}}{6 \pi c^{3}} \ddot{v} v
$$

where the dot indicates differentiation with respect to time $t$. By writing $\ddot{v} v$ as $d(\dot{v} v) / d t-\dot{v}^{2}$ and integrating with respect to $t$, we find

$$
\frac{1}{2} m v^{2}+V(z)=E+\frac{e^{2}}{6 \pi c^{3}} v \dot{v}-\frac{e^{2}}{6 \pi c^{3}} \int_{-\infty}^{t} \dot{v}^{2} d t
$$

where $E$ is the initial energy. By subtracting the equation $\frac{1}{2} m \tilde{v}^{2}+V(\tilde{z})=E$ from Eq. (10) we find to lowest order in $e^{2}$

$$
m \tilde{v} \delta v+V^{\prime}(\tilde{z}) \delta z=\frac{e^{2}}{6 \pi c^{3}} \tilde{v} \frac{d \tilde{v}}{d t}-\frac{e^{2}}{6 \pi c^{3}} \int_{-\infty}^{t} \tilde{a}^{2} d t,
$$

where $\tilde{a}=d \tilde{v} / d t$. By substituting $V^{\prime}(\tilde{z})=-m d \tilde{v} / d t$ in Eq. (11) and dividing by $\tilde{v}^{2}$, we obtain

$$
m \frac{d}{d t}\left(\frac{\delta z}{\tilde{v}}\right)=\frac{e^{2}}{6 \pi \tilde{v} c^{3}} \frac{d \tilde{v}}{d t}-\frac{e^{2}}{6 \pi \tilde{v}^{2} c^{3}} \int_{-\infty}^{t} \tilde{a}^{2} d t .
$$

By integrating this formula from $t=-\infty$ to $t=0$ we have, to second order in $V(z)$,

$$
\left.\delta z\right|_{t=0}=\frac{e^{2}}{6 \pi m c^{3}} v_{f} \log \frac{v_{f}}{v_{i}}-\frac{e^{2}}{6 \pi p c^{3}} I,
$$

where $v_{i}$ and $v_{f}$ are the initial and final velocities, respectively, without radiation, and where

$$
I \equiv \int_{-\infty}^{0}\left\{\int_{-\infty}^{t}\left[\tilde{a}\left(t^{\prime}\right)\right]^{2} d t^{\prime}\right\} d t
$$

We have replaced $m \tilde{v}$ by the final momentum $p=m v_{f}$ in the second term in Eq. (13) because we retain the terms only up to second order in $V(z)$ (and consequently to second order in the acceleration $\tilde{a})$. Integrating by parts and using the assumption that $\tilde{a}(t)=0$ for $t>0$, we find

$$
I=-\int_{-\infty}^{+\infty} t[\tilde{a}(t)]^{2} d t
$$

Now, the power of radiation can be found from Eq. (2) as

$$
P_{r}(t)=\frac{e^{2}}{6 \pi c^{3}}[\tilde{a}(t)]^{2}
$$

in the nonrelativistic approximation. (This is the Larmor formula.) Then the position shift can be expressed as

$$
\left.\delta z\right|_{t=0}=\frac{e^{2}}{6 \pi m c^{3}} v_{f} \log \frac{v_{f}}{v_{i}}+\frac{1}{p} \int_{-\infty}^{+\infty} t P_{r}(t) d t .
$$
by

In terms of the Fourier transform $\hat{a}_{p}(\omega)$ of $\tilde{a}(t)$ defined

$$
\hat{a}_{p}(\omega)=\int_{-\infty}^{+\infty} d t \tilde{a}(t) e^{i \omega t}
$$

we have

$$
I=i \int_{-\infty}^{+\infty} \frac{d \omega}{2 \pi} \hat{a}_{p}(\omega)^{*} \frac{d}{d \omega} \hat{a}_{p}(\omega)
$$

where $A^{*}$ denotes the complex conjugate of $A$. (The subscript " $p$ " in $\hat{a}_{p}(\omega)$ has been inserted to emphasize that this quantity depends on the momentum of the particle. 
This notation will turn out to be useful later.) Thus, to second order in $V(z)$ we have

$$
\begin{aligned}
\left.\delta z\right|_{t=0}= & \frac{e^{2}}{6 \pi m c^{3}} v_{f} \log \frac{v_{f}}{v_{i}} \\
& -\frac{i e^{2}}{6 \pi p c^{3}} \int_{-\infty}^{+\infty} \frac{d \omega}{2 \pi} \hat{a}_{p}(\omega)^{*} \frac{d}{d \omega} \hat{a}_{p}(\omega) .
\end{aligned}
$$

The first term is first-order in the potential because $v_{f}-$ $v_{i}$ is. It is rather puzzling because the position shift is an effect caused by radiation which is second-order in $V(z)$. On the other hand, the second term is clearly secondorder. Note also that the first term would be absent if $v_{f}=v_{i}$, i.e. if $V_{-\infty}=0$. In Sec. IV we will derive the position shift $\left.\delta z\right|_{t=0}$ in the WKB approximation in scalar QED. We will find that the first term in Eq. (20) is absent in the classical limit even if $v_{f} \neq v_{i}$.

\section{THE TRANSITION AMPLITUDE}

We consider a complex scalar field $\psi(t, \mathbf{x})$ which is coupled to the electromagnetic field $A_{\mu}(t, \mathbf{x})$ and accelerated by an external potential $V(z)$ with the properties described in the previous section. This model is given by the following Lagrangian density:

$$
\begin{aligned}
\mathcal{L}= & {\left[\partial_{\mu}-i(e / \hbar c) A_{\mu}\right] \psi^{\dagger} \cdot\left[\partial^{\mu}+i(e / \hbar c) A^{\mu}\right] \psi } \\
& -\frac{1}{\hbar^{2}}\left[m^{2} c^{2}+2 m V(z)\right] \psi^{\dagger} \psi-\frac{1}{4} F_{\mu \nu} F^{\mu \nu}
\end{aligned}
$$

with $\partial_{0} \equiv c^{-1} \partial_{t}$ and $F_{\mu \nu} \equiv \partial_{\mu} A_{\nu}-\partial_{\nu} A_{\mu}$. The mode functions $\Phi(t, \mathbf{x})$ for the free scalar field (with $e=0$ ) satisfy

$$
\left[\partial_{\mu} \partial^{\mu}+m^{2} c^{2} / \hbar^{2}+2 m V(z) / \hbar^{2}\right] \Phi(t, \mathbf{x})=0 .
$$

The energy $p_{0}^{\mathrm{cl}}$ and the momentum $\mathbf{p}^{\mathrm{cl}}$ of the corresponding classical particle satisfy

$$
-\left(p_{0}^{\mathrm{cl}} / c\right)^{2}+\left(\mathbf{p}^{\mathrm{cl}}\right)^{2}+m^{2} c^{2}+2 m V(z)=0 .
$$

(Note that the time component of the 4-momentum $p_{\mu}^{\mathrm{cl}}$ is not $p_{0}^{\mathrm{cl}}$ but $p_{0}^{\mathrm{cl}} / c$.) In the nonrelativistic approximation with $m^{2} c^{2} \gg\left(\mathbf{p}^{\mathrm{cl}}\right)^{2}, 2 m|V(z)|$, we have

$$
p_{0}^{\mathrm{cl}} \approx m c^{2}+\left(\mathbf{p}^{\mathrm{cl}}\right)^{2} / 2 m+V(z) \text {. }
$$

Thus, the particle moves under the influence of the nonrelativistic potential $V(z)$, and the quantum model given by Eq. (21) corresponds to the classical one analyzed in the previous section. Later we will assume $m^{2} c^{2} \gg\left(\mathbf{p}^{\mathrm{cl}}\right)^{2} \gg 2 m|V(z)|$ - this condition implies that the particle is nonrelativistic but the kinetic energy is much larger than the potential energy — but we do not use this assumption for the moment.

The solutions of Eq. (22) which are relevant here can be written as

$$
\Phi_{p, \mathbf{p}_{\perp}}(t, \mathbf{x})=\phi_{p}(z) \exp \left[\frac{i}{\hbar}\left(-p_{0} t+i \mathbf{p}_{\perp} \cdot \mathbf{x}_{\perp}\right)\right],
$$

where $\mathbf{x}_{\perp}=(x, y), \mathbf{p}_{\perp}=\left(p^{x}, p^{y}\right)$ and

$$
p_{0} / c=\sqrt{m^{2} c^{2}+p^{2}+\mathbf{p}_{\perp}^{2}}
$$

with $p>0$. Thus, the quantity $p$ is the $z$-component of the momentum in the region with large and positive $z$ for which the potential $V(z)$ vanishes. The function $\phi_{p}(z)$ satisfies

$$
\left[-\frac{\hbar^{2}}{2 m} \frac{d^{2}}{d z^{2}}+V(z)\right] \phi_{p}(z)=\frac{p^{2}}{2 m} \phi_{p}(z) .
$$

This function can be given in the WKB approximation (see, e.g., Ref. [18]) as

$$
\phi_{p}(z)=\sqrt{\frac{p}{\kappa_{p}(z)}} \exp \left[\frac{i}{\hbar} \int_{0}^{z} \kappa_{p}\left(z^{\prime}\right) d z^{\prime}+g(z)\right]
$$

with

$$
\kappa_{p}(z) \equiv\left[p^{2}-2 m V(z)\right]^{1 / 2}
$$

where $g(z)$ is the correction term of order $\hbar$. We let $g(z)=0$ to work to lowest nontrivial order in $\hbar$. The wave function (28) is normalized so that

$$
\int_{-\infty}^{+\infty} d z \phi_{p^{\prime}}(z)^{*} \phi_{p}(z)=2 \pi \hbar \delta\left(p-p^{\prime}\right)
$$

Strictly speaking, this formula is not correct because the WKB approximation is not valid for some values of $p$. However, since we use only the modes for which the WKB approximation is valid, the final result will not be affected even if we formally use this approximation for all range of $p$, as we do here. Then, the field $\psi$ can be expanded using these modes as

$$
\psi(x)=\hbar c \int \frac{d^{3} \mathbf{p}}{2 p_{0}(2 \pi \hbar)^{3}}\left[A(\mathbf{p}) \Phi_{\mathbf{p}}(x)+B^{\dagger}(\mathbf{p}) \Phi_{\mathbf{p}}(x)^{*}\right],
$$

where $x \equiv(t, \mathbf{x}), \mathbf{p} \equiv\left(p, \mathbf{p}_{\perp}\right)$. The modes $\Phi_{\mathbf{p}}(t, \mathbf{x})$ are orthonormal:

$$
i \hbar \int d^{3} \mathbf{x} \Phi_{\mathbf{p}}(t, \mathbf{x})^{*} \stackrel{\leftrightarrow}{\partial_{t}} \Phi_{\mathbf{p}^{\prime}}(t, \mathbf{x})=2 p_{0}(2 \pi \hbar)^{3} \delta^{3}\left(\mathbf{p}-\mathbf{p}^{\prime}\right)
$$

where $\stackrel{\leftrightarrow}{\partial_{t}} \equiv \overrightarrow{\partial_{t}}-\overleftarrow{\partial_{t}}$. By imposing the usual canonical commutation relations on $\psi(t, \mathbf{x})$,

$$
\begin{aligned}
& {\left[\psi(t, \mathbf{x}), \partial_{t} \psi^{\dagger}\left(t, \mathbf{x}^{\prime}\right)\right]=i \hbar c^{2} \delta^{3}\left(\mathbf{x}-\mathbf{x}^{\prime}\right),} \\
& {\left[\psi^{\dagger}(t, \mathbf{x}), \partial_{t} \psi\left(t, \mathbf{x}^{\prime}\right)\right]=i \hbar c^{2} \delta^{3}\left(\mathbf{x}-\mathbf{x}^{\prime}\right),}
\end{aligned}
$$

with all other equal-time commutators vanishing, one finds

$$
\begin{aligned}
& {\left[A(\mathbf{p}), A^{\dagger}\left(\mathbf{p}^{\prime}\right)\right]=2 p_{0}(2 \pi \hbar)^{3} \delta^{3}\left(\mathbf{p}-\mathbf{p}^{\prime}\right)} \\
& {\left[B(\mathbf{p}), B^{\dagger}\left(\mathbf{p}^{\prime}\right)\right]=2 p_{0}(2 \pi \hbar)^{3} \delta^{3}\left(\mathbf{p}-\mathbf{p}^{\prime}\right)}
\end{aligned}
$$


All other commutators of the creation and annihilation operators vanish. The electromagnetic field in the Feynman gauge can be expanded in the usual manner as

$$
A_{\mu}(t, \mathbf{x})=c \int \frac{d^{3} \mathbf{k}}{2 \omega(2 \pi)^{3}}\left[b_{\mu}(\mathbf{k}) e^{-i \omega t+i \mathbf{k} \cdot \mathbf{x}}+\text { h.c. }\right],
$$

where $\omega=c\|\mathbf{k}\|$. The operators $b_{\mu}(\mathbf{k})$ satisfy

$$
\begin{aligned}
{\left[b_{\mu}(\mathbf{k}), b_{\nu}\left(\mathbf{k}^{\prime}\right)\right] } & =0 \\
{\left[b_{\mu}(\mathbf{k}), b_{\nu}^{\dagger}\left(\mathbf{k}^{\prime}\right)\right] } & =-g_{\mu \nu} 2 \hbar \omega(2 \pi)^{3} \delta^{3}\left(\mathbf{k}-\mathbf{k}^{\prime}\right),
\end{aligned}
$$

where $g_{\mu \nu}$ is the metric of the Minkowski spacetime. Notice that we have used the wave number $\mathbf{k}$ instead of the momentum to label the modes. This is more convenient because the frequency and wave number of the emitted photon are classically well defined, the former being related to how rapidly the acceleration changes. Thus, the momentum of the photon is of order $\hbar$.

We perform our calculations with physical polarization vectors $\epsilon^{(j) \mu}(\mathbf{k}), j=1,2$, of the photon with wave number $\mathbf{k}$, which are real and with vanishing time components. We choose them so that $\epsilon^{(2) z}(\mathbf{k})=0$. It is shown in Appendix A that the calculations using the Fock space with indefinite metric in the Feynman gauge yield the same results. We define the transition amplitude from the initial state consisting of a charged particle with momentum $\mathbf{p} \equiv\left(p, \mathbf{p}_{\perp}\right)$ to the final state consisting of a charged particle with momentum $\mathbf{P} \equiv\left(P, \mathbf{P}_{\perp}\right)$ and a photon with wave number $\mathbf{k}$ and polarization vector $\epsilon^{(j) \mu}(\mathbf{k})$ by

$$
\begin{aligned}
& \mathcal{A}(j, \mathbf{k}, \mathbf{P}, \mathbf{p}) \\
& \equiv \frac{1}{\hbar} \int d^{4} x\left\langle 0\left|b^{(j)}(\mathbf{k}) A(\mathbf{P}) \mathcal{L}_{I}(x) A^{\dagger}(\mathbf{p})\right| 0\right\rangle,
\end{aligned}
$$

where

$$
b^{(j)}(\mathbf{k}) \equiv \epsilon^{(j) \mu}(\mathbf{k}) b_{\mu}(\mathbf{k})
$$

with the following interaction Lagrangian density:

$$
\mathcal{L}_{I}=-\frac{i e}{\hbar c} A^{\mu}\left(\psi^{\dagger} \partial_{\mu} \psi-\partial_{\mu} \psi^{\dagger} \cdot \psi\right)
$$

We only need the transition amplitude with the initial state satisfying $p \gg\left\|\mathbf{p}_{\perp}\right\|$ because we will use a wavepacket state with this condition satisfied. By a straightforward calculation we find

$$
\begin{aligned}
\mathcal{A}(j, \mathbf{k}, \mathbf{P}, \mathbf{p})= & e c^{2} \int d z\left\{\left(\mathbf{p}_{\perp}+\mathbf{P}_{\perp}\right) \cdot \boldsymbol{\epsilon}_{\perp}^{(j)}(\mathbf{k}) \phi_{P}(z)^{*} \phi_{p}(z)\right. \\
- & \left.i \hbar \epsilon^{(j) z}(\mathbf{k}) \phi_{P}(z)^{*} \stackrel{\leftrightarrow}{\partial}_{z} \phi_{p}(z)\right\} e^{-i k_{z} z} \\
& \times(2 \pi \hbar)^{3} \delta^{2}\left(\mathbf{P}_{\perp}+\hbar \mathbf{k}_{\perp}-\mathbf{p}_{\perp}\right) \\
& \times \delta\left(P_{0}+\hbar \omega-p_{0}\right)
\end{aligned}
$$

with $\mathbf{k}_{\perp} \equiv\left(k^{x}, k^{y}\right), \boldsymbol{\epsilon}_{\perp}^{(j)} \equiv\left(\epsilon^{(j) x}, \epsilon^{(j) y}\right)$ and $\stackrel{\leftrightarrow}{\partial}_{z} \equiv \vec{\partial}_{z}$ $-\overleftarrow{\partial}_{z}$. We will calculate this transition amplitude to leading order in $\hbar$. Since we know on physical grounds that the energy and momentum of the photon emitted are of order $\hbar$ and, therefore, much smaller than those of the charged particle, we can assume that $P \gg\left\|\mathbf{P}_{\perp}\right\|$ for the final state because of the assumption that $p \gg\left\|\mathbf{p}_{\perp}\right\|$ for the initial state. Thus, we neglect the term proportional to $\left(\mathbf{p}_{\perp}+\mathbf{P}_{\perp}\right) \cdot \boldsymbol{\epsilon}_{\perp}^{(j)}(\mathbf{k})$ in Eq. (40). Then, to lowest nontrivial order in $\hbar$ we find

$$
\begin{aligned}
\mathcal{A}(j, \mathbf{k}, \mathbf{P}, \mathbf{p})= & e c^{2} \epsilon^{(j) z}(\mathbf{k}) G\left(k_{z}, P, p\right) \\
& \times(2 \pi \hbar)^{3} \delta^{2}\left(\mathbf{P}_{\perp}+\hbar \mathbf{k}_{\perp}-\mathbf{p}_{\perp}\right) \\
& \times \delta\left(P_{0}+\hbar \omega-p_{0}\right),
\end{aligned}
$$

where

$$
G\left(k_{z}, P, p\right) \equiv \int d z \phi_{P}(z)^{*}\left[\kappa_{p}(z)+\kappa_{P}(z)\right] \phi_{p}(z) e^{-i k_{z} z}
$$

Next we evaluate the integral $G\left(k_{z}, P, p\right)$ to lowest nontrivial order in $\hbar$. We keep only the terms which are firstorder in $V(z)$ because $G\left(k_{z}, P, p\right)$ will be squared in the position shift for which we need only the second-order terms. First we note that to first order in $V(z)$ we can write

$$
G\left(k_{z}, P, p\right)=2 \sqrt{P p} \int d z \exp \left\{i \int_{0}^{z} K\left(z^{\prime}\right) d z^{\prime}\right\},
$$

where

$$
K(z) \equiv\left[\kappa_{p}(z)-\kappa_{P}(z)\right] / \hbar-k_{z}
$$

This follows from the fact that $\left[\kappa_{p}(z) / \kappa_{P}(z)\right]^{1 / 2}+$ $\left[\kappa_{P}(z) / \kappa_{p}(z)\right]^{1 / 2}-2$ is second-order in $V(z)$. Now, for any function $g(z)$, one has

$$
\int_{a}^{b} d z e^{i g(z)}=-i\left[\frac{e^{i g(z)}}{g^{\prime}(z)}\right]_{a}^{b}-i \int_{a}^{b} d z \frac{g^{\prime \prime}(z)}{\left[g^{\prime}(z)\right]^{2}} e^{i g(z)} .
$$

We use this formula with $g(z)=\int_{0}^{z} K(z) d z+i \epsilon|z|$, where the term $i \epsilon|z|$ with $\epsilon>0$ has been inserted to regularize the integral. The boundary terms vanish because one cannot have $K(z)=0$ as well as

$$
\begin{gathered}
p_{0}=P_{0}+\hbar \omega, \\
\mathbf{p}_{\perp}=\mathbf{P}_{\perp}+\hbar \mathbf{k}_{\perp},
\end{gathered}
$$

which are enforced by the $\delta$-functions in Eq. (41), without violating the mass-shell conditions. Hence we have

$$
\begin{array}{rl}
G\left(k_{z}, P, p\right)=-2 & i \sqrt{P p} \int_{-\infty}^{+\infty} d z \frac{K^{\prime}(z)}{[K(z)]^{2}} \\
& \times \exp \left[i \int_{0}^{z} K\left(z^{\prime}\right) d z^{\prime}\right] .
\end{array}
$$

Let us find a small $\hbar$ approximation for $K(z)$. By squaring the energy-conservation equation (46a), we find

$$
p^{2}+\mathbf{p}_{\perp}^{2} \approx P^{2}+\mathbf{P}_{\perp}^{2}+2 m \hbar \omega
$$


in the nonrelativistic approximation and to first order in $\hbar$. By using the inequality

$$
\left|\mathbf{p}_{\perp}^{2}-\mathbf{P}_{\perp}^{2}\right| \leq\left\|\mathbf{p}_{\perp}-\mathbf{P}_{\perp}\right\|\left\|\mathbf{p}_{\perp}+\mathbf{P}_{\perp}\right\|
$$

and Eq. 46b), we find

$$
\left|\mathbf{p}_{\perp}^{2}-\mathbf{P}_{\perp}^{2}\right| \leq \hbar \omega / c \cdot\left\|\mathbf{p}_{\perp}+\mathbf{P}_{\perp}\right\| \ll m \hbar \omega .
$$

Using this in Eq. (48) we have

$$
p^{2}-P^{2} \approx 2 m \hbar \omega .
$$

Hence we obtain the following approximate formula:

$$
\kappa_{p}(z)-\kappa_{P}(z) \approx \frac{\partial \kappa_{P}(z)}{\partial P^{2}}\left(p^{2}-P^{2}\right) \approx \frac{\hbar \omega}{v_{p}(z)},
$$

where

$$
v_{p}(z) \equiv \kappa_{p}(z) / m=\sqrt{p^{2}-2 m V(z)} / m
$$

is the speed of the corresponding classical particle. Hence,

$$
K(z) \approx \frac{\omega}{v_{p}(z)}-k_{z} .
$$

Furthermore, since the charged particle is nonrelativistic, we have $\omega / v_{p}(z)=c\|\mathbf{k}\| / v_{p}(z) \gg k_{z}$. Thus,

$$
K(z) \approx \frac{\omega}{v_{p}(z)} .
$$

By substituting Eq. (55) in Eq. (47) and letting $\sqrt{P p} \approx$ $p$, we obtain

$$
\begin{aligned}
& G\left(k_{z}, P, p\right) \\
& \approx \frac{2 i p}{\omega} \int_{-\infty}^{+\infty} d z v_{p}^{\prime}(z) \exp \left\{i \omega \int_{0}^{z}\left[v_{p}\left(z^{\prime}\right)\right]^{-1} d z^{\prime}\right\} .
\end{aligned}
$$

Let us define the function $T(z)$ by

$$
T \equiv \int_{0}^{z}\left[v_{p}\left(z^{\prime}\right)\right]^{-1} d z^{\prime}+T_{0},
$$

where $T_{0}$ is a constant. Then by using

$$
d z v_{p}^{\prime}(z)=d z \frac{d v_{p}}{d z}=d T \frac{d v_{p}}{d T}
$$

and noting that $T \rightarrow \pm \infty$ as $z \rightarrow \pm \infty$ because $v_{p}(z)$ is always positive, we obtain

$$
G\left(k_{z}, P, p\right)=\frac{2 i p}{\omega} \int_{-\infty}^{+\infty} d T \frac{d v_{p}}{d T} e^{i \omega\left(T-T_{0}\right)} .
$$

Now, Eq. (57) implies that $d z / d T=v_{p}$. Thus, the quantity $T$ can be identified with the time coordinate of the classical particle if the constant $T_{0}$ is chosen appropriately. Recall that we need to evaluate $G\left(k_{z}, P, p\right)$ only to first order in $V(z)$. Since the acceleration $d v_{p} / d T$ is firstorder in $V(z)$, we need to find $T_{0}$ only to zeroth order in $V(z)$, i.e. we may neglect the potential. Since $T=T_{0}$ for $z=0$, the constant $T_{0}$ is the time when the particle is at $z=0$. Letting the particle be at $z=z_{0}$ for $T=0$, we have $z_{0} / T_{0}=-v_{p}=-p / m$. Hence $T_{0}=-m z_{0} / p$. By substituting this in Eq. (59) we find

$$
G\left(k_{z}, P, p\right)=\frac{2 i p}{\omega} \hat{a}_{p}(\omega) \exp \left(i m \omega z_{0} / P\right),
$$

where the Fourier transform of the acceleration, $\hat{a}_{p}(\omega)$, is defined by Eq. (18) [with the identification $d v_{p} / d T=$ $\tilde{a}(t)]$. One may replace $p$ by $P$ and vice versa in Eq. (60) because the difference $p-P$ is of order $\hbar$.

It is instructive to compare this result with the transition amplitude for radiation due to a classical current with the following interaction Lagrangian density:

$$
\mathcal{L}_{I \mathrm{cl}}=(e / c) A_{\mu}(t, \mathbf{x}) j^{\mu}(t, \mathbf{x})
$$

Here, the current density $j^{\mu}(x)$ is given by

$$
\begin{aligned}
& j^{0}(t, \mathbf{x})=c \delta^{2}\left(\mathbf{x}_{\perp}\right) \delta[z-z(t)], \\
& j^{z}(t, \mathbf{x})=v(t) \delta^{2}\left(\mathbf{x}_{\perp}\right) \delta[z-z(t)]
\end{aligned}
$$

with $j^{x}(t, \mathbf{x})=j^{y}(t, \mathbf{x})=0$, where $z(t)$ is the position of the charged particle at time $t$ and where $v(t)=z^{\prime}(t)$ is its velocity in the $z$-direction. The transition amplitude from the zero-photon state $|0\rangle_{\gamma}$ to a one-photon state $b^{(j) \dagger}(\mathbf{k})|0\rangle_{\gamma}$ is

$$
\begin{aligned}
\mathcal{A}_{\mathrm{cl}}(j, \mathbf{k}) & \equiv \frac{1}{\hbar} \int d^{4} x_{\gamma}\left\langle 0\left|b^{(j)}(\mathbf{k}) \mathcal{L}_{I \mathrm{cl}}(x)\right| 0\right\rangle_{\gamma} \\
& =e \epsilon^{(j) z}(\mathbf{k}) \int_{-\infty}^{+\infty} d t v(t) \exp \left[i \omega t-i k_{z} z(t)\right] .
\end{aligned}
$$

By integrating by parts with a damping factor $e^{-\epsilon|t|}$ and dropping the surface terms because $\omega-k_{z} v(z) \neq 0$ for all $z$, we obtain

$$
\mathcal{A}_{\mathrm{cl}}(j, \mathbf{k})=i e \epsilon^{(j) z}(\mathbf{k}) \omega \int_{-\infty}^{+\infty} d t \frac{v^{\prime}(t) \exp \left[i \omega t-i k_{z} z(t)\right]}{\left[\omega-k_{z} v(t)\right]^{2}} .
$$

Since $\omega=c\|\mathbf{k}\| \gg k_{z} v(t)$ and $\omega t \gg k_{z} z(t)$, we find

$$
\begin{aligned}
\mathcal{A}_{\mathrm{cl}}(j, \mathbf{k}) & \approx \frac{i e \epsilon^{(j) z}(\mathbf{k})}{\omega} \int_{-\infty}^{+\infty} d t v^{\prime}(t) e^{i \omega t} \\
& =\frac{i e \epsilon^{(j) z}(\mathbf{k})}{\omega} \hat{a}_{p}(\omega) .
\end{aligned}
$$

Notice the similarity of this expression with Eq. (60). Our result obtained in the small $\hbar$ approximation is closely related to the emission process by the corresponding classical particle. 


\section{THE POSITION SHIFT IN QUANTUM ELECTRODYNAMICS} by

Now, let us consider an initial wave-packet state given

$$
|i\rangle=\int \frac{d^{3} \mathbf{p}}{\sqrt{2 p_{0}}(2 \pi \hbar)^{3}} f(\mathbf{p}) A^{\dagger}(\mathbf{p})|0\rangle .
$$

The normalization condition $\langle i \mid i\rangle=1$ leads to

$$
\int \frac{d^{3} \mathbf{p}}{(2 \pi \hbar)^{3}}|f(\mathbf{p})|^{2}=1
$$

The function $f(\mathbf{p})$ will be identified later as the nonrelativistic one-particle wave function in the momentum representation. We choose the following form:

$$
f(\mathbf{p})=|f(\mathbf{p})| e^{-i p z_{0} / \hbar}
$$

with

$$
|f(\mathbf{p})|=N \exp \left[-\frac{(p-\bar{p})^{2}+\left\|\mathbf{p}_{\perp}\right\|^{2}}{2(\Delta p)^{2}}\right],
$$

where $N$ is the normalization constant. Here, $\Delta p$ and $\bar{p}(>0)$ are the width of the wave packet in the momentum space and the average momentum [in the region with $V(z)=0]$, respectively. The constant $z_{0}$ will turn out to be the average $z$-coordinate at $t=0$. We now assume that $m^{2} c^{2} \gg \bar{p}^{2} \gg m|V(z)|$. We also assume that $\Delta p \ll \bar{p}$. The transverse momentum $\mathbf{p}_{\perp}$ is much smaller than $\bar{p}$ under this assumption. This wave packet describes a nonrelativistic charged particle moving in the positive $z$-direction with its kinetic energy much larger than $|V(z)|$. The initial state $|i\rangle$ given by Eq. (66) leads to the following one-photon final state according to the standard time-dependent perturbation theory in the interaction picture:

$$
\begin{aligned}
|f, 1 \gamma\rangle= & i \sum_{j=1}^{2} \int \frac{d^{3} \mathbf{k}}{2 \hbar \omega(2 \pi)^{3}} \int \frac{d^{3} \mathbf{P}}{2 P_{0}(2 \pi \hbar)^{3}} \\
& \times \int \frac{d^{3} \mathbf{p}}{\sqrt{2 p_{0}}(2 \pi \hbar)^{3}} f(\mathbf{p}) \mathcal{A}(j, \mathbf{k}, \mathbf{P}, \mathbf{p}) \\
& \times b^{(j) \dagger}(\mathbf{k}) A^{\dagger}(\mathbf{P})|0\rangle
\end{aligned}
$$

where $P_{0} / c \equiv \sqrt{m^{2} c^{2}+P^{2}+\mathbf{P}_{\perp}^{2}}$. The amplitude $\mathcal{A}(j, \mathbf{k}, \mathbf{P}, \mathbf{p})$ is defined by Eq. (411) with $G\left(k_{z}, P, p\right)$ given by Eq. (60). By substituting Eq. (41) in Eq. (70) and using, for any function $F(\mathbf{p})$,

$$
\begin{aligned}
& \int \frac{d^{3} \mathbf{p}}{\sqrt{2 p_{0}}} F(\mathbf{p}) \delta^{2}\left(\mathbf{p}_{\perp}+\hbar \mathbf{k}_{\perp}-\mathbf{P}_{\perp}\right) \delta\left(P_{0}+\hbar \omega-p_{0}\right) \\
& =\frac{\sqrt{2 p_{0}}}{2 p c^{2}} F(\mathbf{p})
\end{aligned}
$$

where $\mathbf{p}$ on the right-hand side is expressed in terms of $\mathbf{P}$ and $\mathbf{k}$ through the relations (46), and recalling that $\epsilon^{(2) z}(\mathbf{k})=0$, we obtain

$$
\begin{aligned}
|f, 1 \gamma\rangle= & \frac{i e}{\hbar} \int \frac{d^{3} \mathbf{k}}{2 \omega(2 \pi)^{3}} \int \frac{d^{3} \mathbf{P}}{2 P_{0}(2 \pi \hbar)^{3}} \frac{\sqrt{2 p_{0}}}{2 p} f(\mathbf{p}) \\
& \times \epsilon^{(1) z}(\mathbf{k}) G\left(k_{z}, P, p\right) b^{(1) \dagger}(\mathbf{k}) A^{\dagger}(\mathbf{P})|0\rangle .
\end{aligned}
$$

Now, from Eq. (51) we have $p-P \approx m \hbar \omega / P$. By using this approximation in Eq. (68) we find

$$
f(\mathbf{p}) \approx|f(\mathbf{p})| \exp \left(-i P z_{0} / \hbar-i m \omega z_{0} / P\right) .
$$

By substituting this equation and Eq. (60) in Eq. (72) we obtain

$$
\begin{aligned}
|f, 1 \gamma\rangle= & -\frac{e}{\hbar} \int \frac{d^{3} \mathbf{k}}{2 \omega^{2}(2 \pi)^{3}} \int \frac{d^{3} \mathbf{P}}{\sqrt{2 P_{0}}(2 \pi \hbar)^{3}} \\
& \times|f(\mathbf{p})| \exp \left(-i P z_{0} / \hbar\right) \epsilon^{(1) z}(\mathbf{k}) \hat{a}_{p}(\omega) \\
& \times b^{(1) \dagger}(\mathbf{k}) A^{\dagger}(\mathbf{P})|0\rangle
\end{aligned}
$$

where

$$
\mathbf{p}=\left(p, \mathbf{p}_{\perp}\right)=\left(P+m \hbar \omega / P, \mathbf{P}_{\perp}+\hbar \mathbf{k}_{\perp}\right) .
$$

We have approximated $p_{0}$ by $P_{0}$ on the right-hand side of Eq. (74).

The emission probability can be obtained to leading order in $\hbar$ from Eq. (74) as

$$
\begin{aligned}
\mathcal{P}_{r} \equiv & \langle f, 1 \gamma \mid f, 1 \gamma\rangle \\
= & \frac{e^{2}}{\hbar} \int \frac{d^{3} \mathbf{k}}{2 \omega^{3}(2 \pi)^{3}} \int \frac{d^{3} \mathbf{P}}{(2 \pi \hbar)^{3}} \\
& \quad \times|f(\mathbf{p})|^{2}\left[\epsilon^{(1) z}(\mathbf{k})\right]^{2}\left|\hat{a}_{p}(\omega)\right|^{2} \\
\approx & \frac{e^{2}}{\hbar} \int \frac{d^{3} \mathbf{k}}{2 \omega^{3}(2 \pi)^{3}}\left[\epsilon^{(1) z}(\mathbf{k})\right]^{2}\left|\hat{a}_{\bar{p}}(\omega)\right|^{2},
\end{aligned}
$$

where we have used the fact that the function $|f(\mathbf{p})|$ is sharply peaked about $\left(p, \mathbf{p}_{\perp}\right)=(\bar{p}, 0)$. Recalling that $d^{3} \mathbf{k}=d \Omega_{\mathbf{k}} \omega^{2} d \omega / c^{3}$, where $d \Omega_{\mathbf{k}}$ is the solid-angle element in the $\mathbf{k}$ space, and that the average of $\left|\epsilon^{(j) z}(\mathbf{k})\right|^{2}$ for each value of $\omega$ is $2 / 3$, we find

$$
\mathcal{P}_{r}=\frac{e^{2}}{3 \pi \hbar c^{3}} \int_{0}^{\infty} \frac{d \omega}{2 \pi \omega}\left|\hat{a}_{\bar{p}}(\omega)\right|^{2} .
$$

Notice that this probability is infrared divergent unless $\hat{a}_{\bar{p}}(0)=V_{-\infty} / \bar{p}=0$. However, the expected energy of the photon is finite and is given by

$$
\begin{aligned}
E_{r} & =\frac{e^{2}}{3 \pi \hbar c^{3}} \int_{0}^{\infty} \frac{d \omega}{2 \pi \omega} \hbar \omega\left|\hat{a}_{\bar{p}}(\omega)\right|^{2} \\
& =\frac{e^{2}}{6 \pi c^{3}} \int_{-\infty}^{+\infty} d t\left[a_{\bar{p}}(t)\right]^{2},
\end{aligned}
$$

where $a_{\bar{p}}(t)$ is the acceleration of the corresponding classical particle at time $t$. This is nothing but the Larmor formula (16). 
For the classical current discussed at the end of the previous section, the one-photon final state is

$$
\begin{aligned}
|f, 1 \gamma\rangle_{\mathrm{cl}} & =\frac{i}{\hbar} \int \frac{d^{3} \mathbf{k}}{2 \omega(2 \pi)^{3}} \mathcal{A}_{\mathrm{cl}}(1, \mathbf{k}) b^{(1) \dagger}(\mathbf{k})|0\rangle_{\gamma} \\
& =-\frac{e}{\hbar} \int \frac{d^{3} \mathbf{k}}{2 \omega^{2}(2 \pi)^{3}} \epsilon^{(1) z}(\mathbf{k}) \hat{a}_{p}(\omega) b^{(1) \dagger}(\mathbf{k})|0\rangle_{\gamma}
\end{aligned}
$$

The emission probability calculated from this state agrees with Eq. (77).

Now, we derive the quantum expression of the position shift to order $e^{2}$ in the small $\hbar$ approximation and compare it with the result in the Lorentz-Dirac theory derived in Sec. III We assume that the constant $z_{0}$ in Eq. (68) is larger than any $z$ with $V(z) \neq 0$ and that the wave packet is far into the region with $V(z)=0$ at $t=0$. First we note that the expected value of the $z$-coordinate at $t=0$ with the electromagnetic field turned off is given by

$$
\langle z\rangle_{t=0}^{\mathrm{off}}=\frac{i \hbar}{2} \int \frac{d^{3} \mathbf{p}}{(2 \pi \hbar)^{3}}\left[f(\mathbf{p})^{*} \frac{\partial f(\mathbf{p})}{\partial p}-\frac{\partial f(\mathbf{p})^{*}}{\partial p} f(\mathbf{p})\right],
$$

where $f(\mathbf{p})$ is defined by Eq. (68). This formula can be understood as the statement that the function $f(\mathbf{p})$ is the momentum representation of the one-particle wave function. The derivation of Eq. (80) is given in Appendix B. Since

$$
\frac{i \hbar}{2}\left[f(\mathbf{p})^{*} \frac{\partial f(\mathbf{p})}{\partial p}-\frac{\partial f(\mathbf{p})^{*}}{\partial p} f(\mathbf{p})\right]=z_{0}|f(\mathbf{p})|^{2},
$$

we find

$$
\langle z\rangle_{t=0}^{\mathrm{off}}=z_{0}
$$

Next we calculate the expected value of the $z$ coordinate at $t=0$ with the electromagnetic field turned on. At lowest nontrivial order in $e$, the final state can be given as

$$
|f\rangle=|f, 0 \gamma\rangle+|f, 1 \gamma\rangle
$$

where $|f, 1 \gamma\rangle$ is the one-photon final state given by Eq. (74) and where the zero-photon final state $|f, 0 \gamma\rangle$ can be written as

$$
|f, 0 \gamma\rangle=\int \frac{d^{3} \mathbf{p}}{\sqrt{2 p^{0}}(2 \pi \hbar)^{3}}[1+i \mathcal{F}(\mathbf{p})] f(\mathbf{p}) A^{\dagger}(\mathbf{p})|0\rangle .
$$

We will not explicitly evaluate the one-loop forwardscattering amplitude $\mathcal{F}(\mathbf{p})$, which is of order $e^{2}$. The expected value of the $z$-coordinate at $t=0$ is the sum of the zero-photon and one-photon contributions. The zero-photon contribution, $\langle z\rangle_{t=0}^{(0)}$, can be obtained by replacing $f(\mathbf{p})$ by $[1+i \mathcal{F}(\mathbf{p})] f(\mathbf{p})$ in Eq. (80). The result is

$$
\langle z\rangle_{t=0}^{(0)}=\int \frac{d^{3} \mathbf{p}}{(2 \pi \hbar)^{3}}\left|f_{\mathbf{p}}\right|^{2}\left[z_{0}(1-2 \operatorname{Im} \mathcal{F})-\hbar \frac{\partial}{\partial p} \operatorname{Re} \mathcal{F}\right],
$$

where we have written $f(\mathbf{p}) \equiv f_{\mathbf{p}}$ and used Eq. (81). The contribution from the one-photon state, $\langle z\rangle_{t=0}^{(1)}$, is similar except that one needs to trace over the photon states. Recall that we have calculated $G\left(k_{z}, P, p\right)$ in Eq. (60) to lowest order in $\hbar$. We define $\hat{a}_{1}(\mathbf{k}, \mathbf{p})$ as a quantity such that the expression for $G\left(k_{z}, P, p\right)$ in that equation will be valid to next order in $\hbar$ if $\epsilon^{(1) z}(\mathbf{k}) \hat{a}_{p}(\omega)$ is replaced by it. (Introduction of $\hat{a}_{1}(\mathbf{k}, \mathbf{p})$ is necessary because $\langle z\rangle_{t=0}^{(0)}$ and $\langle z\rangle_{t=0}^{(1)}$ are of order $\hbar^{-1}$.) Then, we have, to order $\hbar^{0}$,

$$
\begin{aligned}
\langle z\rangle_{t=0}^{(1)}= & \frac{i e^{2}}{2} \int \frac{d^{3} \mathbf{k}}{2 \omega^{3}(2 \pi)^{3}} \int \frac{d^{3} \mathbf{P}}{(2 \pi \hbar)^{3}} \\
& \times\left[\left|\hat{a}_{1}(\mathbf{k}, \mathbf{p})\right|^{2}\left(f_{\mathbf{p}}^{*} \frac{\partial f_{\mathbf{p}}}{\partial P}-\frac{\partial f_{\mathbf{p}}^{*}}{\partial P} f_{\mathbf{p}}\right)\right. \\
& +\left|\epsilon^{(1) z}(\mathbf{k})\right|^{2}\left|f_{\mathbf{p}}\right|^{2} \\
& \left.\times\left(\hat{a}_{p}(\omega)^{*} \frac{\partial \hat{a}_{p}(\omega)}{\partial P}-\frac{\partial \hat{a}_{p}(\omega)^{*}}{\partial P} \hat{a}_{p}(\omega)\right)\right],
\end{aligned}
$$

where $\mathbf{p}$ is related to $\mathbf{P}$ by Eq. (75). The integration variables $\mathbf{P}$ can be changed to $\mathbf{p}$ if we change the derivative $\partial / \partial P$ to $\partial / \partial p$ at the same time. Then by using Eq. (81) again we find

$$
\begin{aligned}
\langle z\rangle_{t=0}^{(1)}= & \int \frac{d^{3} \mathbf{p}\left|f_{\mathbf{p}}\right|^{2}}{(2 \pi \hbar)^{3}} \int \frac{d^{3} \mathbf{k}}{2 \omega^{3}(2 \pi)^{3}} \\
& \times\left[\frac{e^{2} z_{0}}{\hbar}\left|\hat{a}_{1}(\mathbf{k}, \mathbf{p})\right|^{2}+\frac{i e^{2}}{2}\left|\epsilon^{(1) z}(\mathbf{k})\right|^{2}\right. \\
& \left.\times\left(\hat{a}_{p}(\omega)^{*} \frac{\partial \hat{a}_{p}(\omega)}{\partial p}-\frac{\partial \hat{a}_{p}(\omega)^{*}}{\partial p} \hat{a}_{p}(\omega)\right)\right],
\end{aligned}
$$

Unitarity implies that $\langle f, 0 \gamma \mid f, 0 \gamma\rangle+\langle f, 1 \gamma \mid f, 1 \gamma\rangle=1$ at order $e^{2}$. This results in the following relation:

$$
2 \operatorname{Im} \mathcal{F}=\frac{e^{2}}{\hbar} \int \frac{d^{3} \mathbf{k}}{2 \omega^{3}(2 \pi)^{3}}\left|\hat{a}_{1}(\mathbf{k}, \mathbf{p})\right|^{2} .
$$

Both sides are equal to the emission probability, which is given by Eq. (77) at leading order in $\hbar$. By adding $\langle z\rangle_{t=0}^{(0)}$ and $\langle z\rangle_{t=0}^{(1)}$ given by Eqs. (85) and (86), respectively, using Eq. (88) and recalling that the function $f_{\mathbf{p}}$ is sharply peaked about $(p, \mathbf{p})=(\bar{p}, 0)$ we have

$$
\begin{aligned}
\langle z\rangle_{t=0} \approx & z_{0}-\left.\hbar \frac{\partial}{\partial p} \operatorname{Re} \mathcal{F}\right|_{p=\bar{p}, \mathbf{p}_{\perp}=0} \\
& +\frac{i e^{2}}{2} \int \frac{d^{3} \mathbf{k}\left|\epsilon^{(1) z}(\mathbf{k})\right|^{2}}{2 \omega^{3}(2 \pi)^{3}} \\
& \times\left[\hat{a}_{p}(\omega)^{*} \frac{\partial}{\partial p} \hat{a}_{p}(\omega)-\frac{\partial}{\partial p} \hat{a}_{p}(\omega)^{*} \cdot \hat{a}_{p}(\omega)\right]_{p=\bar{p}}
\end{aligned}
$$

Now we note that the classical acceleration depends on $p$ and $t$ through $z=p t / m+z_{0}$. [One can use the freeparticle approximation here because the last term in Eq. 
(89), where $\hat{a}_{p}(\omega)$ appears, is already second-order in $V(z)$.] Thus,

$$
\left(t \frac{\partial}{\partial t}-p \frac{\partial}{\partial p}\right) \int_{-\infty}^{+\infty} \frac{d \omega}{2 \pi} \hat{a}_{p}(\omega) e^{-i \omega t}=0 .
$$

Then we find

$$
\frac{\partial}{\partial p} \hat{a}_{p}(\omega)=-\frac{1}{p} \hat{a}_{p}(\omega)-\frac{\omega}{p} \frac{\partial}{\partial \omega} \hat{a}_{p}(\omega) .
$$

From this formula and the fact that $\hat{a}_{p}(-\omega)=\hat{a}_{p}(\omega)^{*}$ [because the acceleration is real], we obtain

$$
\begin{aligned}
\langle z\rangle_{t=0} \approx & z_{0}-\left.\hbar \frac{\partial}{\partial p} \operatorname{Re} \mathcal{F}\right|_{p=\bar{p}, \mathbf{p}_{\perp}=0} \\
& -\frac{i e^{2}}{6 \pi p c^{3}} \int_{-\infty}^{+\infty} \frac{d \omega}{2 \pi} \hat{a}_{\bar{p}}(\omega)^{*} \frac{\partial}{\partial \omega} \hat{a}_{\bar{p}}(\omega) .
\end{aligned}
$$

The forward-scattering amplitude $\mathcal{F}$ arises in the oneloop diagram without photon emission. Therefore, the second term in Eq. (92) comes from the quantum correction to the potential rather than from the reaction to radiation. (We will discuss this term in the next section.) Thus, the position shift due to radiation can be given as

$$
\begin{aligned}
\left.\delta z\right|_{t=0} ^{\text {Quantum }} & =-\frac{i e^{2}}{6 \pi p c^{3}} \int_{-\infty}^{+\infty} \frac{d \omega}{2 \pi} \hat{a}_{\bar{p}}(\omega)^{*} \frac{\partial}{\partial \omega} \hat{a}_{\bar{p}}(\omega) \\
& =\frac{1}{\bar{p}} \int_{-\infty}^{+\infty} d t t P_{r}(t)
\end{aligned}
$$

where $P_{r}(t)$ given by Eq. (16) is the power radiated.

One can readily see that Eq. (93) does not agree with Eq. (20) derived in the Lorentz-Dirac theory. However, these equations would be identical if we removed the first term proportional to $\log \left(v_{f} / v_{i}\right)$ from Eq. (20). This term can be traced back to the term proportional to $v \dot{v}$ in Eq. (10) which has arisen due to the substitution $\ddot{v} v=d(\dot{v} v) / d t-\dot{v}^{2}$. Therefore, Eq. (93) is reproduced in the classical theory by modifying the energy-conservation equation (9) as

$$
\frac{d}{d t}\left[\frac{1}{2} m v^{2}+V(z)\right]=-\frac{e^{2}}{6 \pi c^{3}} \dot{v}^{2}=-P_{r}(t) .
$$

This formula can be interpreted as stating that the energy of the charged particle is lost through radiation at each time. In other words, the $\hbar \rightarrow 0$ limit of scalar QED at order $e^{2}$ can be reproduced by classical electrodynamics if momentum conservation is disregarded and energy conservation is used at each moment of time, as far as the position shift is concerned. This seems reasonable because in the quantum theory the energy is conserved in the rest frame determined by the potential $V(z)$ but the momentum is not conserved since the potential is $z$-dependent.

\section{SOME COMMENTS ON THE FORWARD-SCATTERING AMPLITUDE}

The imaginary part $\operatorname{Im} \mathcal{F}$ of the forward-scattering amplitude is related to the photon emission probability by unitarity, as we mentioned before. The real part $\operatorname{Re} \mathcal{F}$ represents the quantum correction to the potential (which may be nonlocal). To illustrate this fact let us compute the forward-scattering amplitude due to a slight change in the potential in the form $V(z) \rightarrow V(z)+\delta V(z)$, with $\delta V(z)$ regarded as perturbation. We find

$$
\begin{aligned}
\mathcal{F} & =-\frac{2 m c^{2}}{\hbar} \int \frac{d^{3} \mathbf{P}}{2 P_{0}(2 \pi \hbar)^{3}} \int d^{4} x \Phi_{\mathbf{P}}^{*} \delta V(z) \Phi_{\mathbf{p}} \\
& =-\frac{m}{\hbar p} \int_{-\infty}^{+\infty} d z \delta V(z)
\end{aligned}
$$

to lowest order in $V(z)$ and $\delta V(z)$. The position shift due to this change in the potential is

$$
-\hbar \frac{\partial}{\partial p} \operatorname{Re} \mathcal{F}=-\frac{m}{p^{2}} \int_{-\infty}^{+\infty} d z \delta V(z) .
$$

It can readily be verified that the position shift is given by the same formula in the classical theory. We have not been able to find $\operatorname{Re} \mathcal{F}$ to order $\hbar^{0}$ in our model but will show that the quantum correction to the potential is of order $\hbar^{-1}$. This will imply that the electromagnetic correction to the potential at order $e^{2}$ cannot be obtained in the classical theory.

Since the potential is part of the mass term in our model, its quantum correction is related to the mass renormalization. If we let $V(z)=0$ in the mass function given by

$$
\left[m^{2} c^{2}+2 m V(z)\right] / \hbar^{2} \equiv[m(z)]^{2} c^{2} / \hbar^{2},
$$

its quantum correction can be computed in the dimensional regularization with $D=4-2 \epsilon$ as

$$
\delta m^{2}=-\frac{e^{2}}{16 \pi^{2} \hbar c} m^{2}\left(\frac{3}{\epsilon}-3 \gamma+7-3 \ln \frac{m^{2}}{4 \pi \mu^{2}}\right),
$$

where $\mu$ is the renormalization scale and $\gamma$ is Euler's constant. The mass function is corrected according to this formula for large and positive $z$ for which $V(z)=0$. For large and negative $z$, the squared mass is $m^{2}+$ $2 m V_{-\infty} / c^{2}$. Therefore the quantum correction to the mass term is obtained by replacing $m^{2}$ by this value in Eq. (98). This implies that the difference between the quantum corrections to the potential at $z= \pm \infty$ is of order $\hbar^{-1}$. Hence, this correction cannot be obtained in classical electrodynamics 21].

If the potential is slowly varying so that the WKB approximation is valid, it is reasonable to expect that the quantum correction to the mass term at leading order in $\hbar$ is obtained by replacing $m^{2}$ by $[m(z)]^{2}$ in Eq. (98) for all $z$. Then,

$$
\delta[m(z)]^{2}=-\frac{e^{2}[m(z)]^{2}}{16 \pi^{2} \hbar c}\left(\frac{3}{\epsilon}-3 \gamma+7-3 \ln \frac{[m(z)]^{2}}{4 \pi \mu^{2}}\right) .
$$


The quantum correction to the potential can be expressed by using this formula and Eq. (98) as

$$
\begin{aligned}
\delta\left(\frac{V(z)}{m c^{2}}\right) & =\frac{1}{2} \delta\left(\frac{[m(z)]^{2}}{m^{2}}\right) \\
& =\frac{3 e^{2}}{32 \pi^{2} \hbar c} \frac{[m(z)]^{2}}{m^{2}} \ln \frac{[m(z)]^{2}}{m^{2}}
\end{aligned}
$$

to lowest order in $e^{2}$. Since the acceleration is $-V^{\prime}(z) / m$, this formula shows that the correction to the acceleration is ultraviolet finite at leading order in $\hbar$.

\section{CONCLUSION}

In this paper we studied a wave packet of a charged scalar particle moving in the $z$-direction and accelerated by a potential which depends only on $z$. Our main conclusion is that, in the limit $\hbar \rightarrow 0$, the position shift due to the reaction to radiation agrees not with the formula in the Lorentz-Dirac theory but with that obtained by assuming that the kinetic energy is lost to radiation according to the Larmor formula at each moment of time, at leading order in $e^{2}$ and in the nonrelativistic approximation. It will be interesting to study a more general system and see if the result obtained here can be generalized.

Finally, let us discuss the magnitude of the position shift in comparison with the width of the wave packet. As we have seen in the introduction, the position shift may be much smaller than indicated by the fact that it is of order $\hbar^{0}$. First we note that the first term in Eq. (17), which represents the discrepancy between the LorentzDirac theory and the quantum theory, is in fact much smaller than the Compton wavelength of the particle:

$$
\frac{e^{2}}{6 \pi m c^{3}} v_{f} \log \frac{v_{f}}{v_{i}}=\frac{2 \alpha \hbar}{3 m c}\left(\frac{v_{f}}{c}\right) \log \frac{v_{f}}{v_{i}} \ll \frac{\hbar}{m c} .
$$

Thus, it is much smaller than the width of any nonrelativistic wave packet. Hence, this term would be practically unobservable even if it was present. The second term, which is the position shift in the quantum theory, grows linearly as a function of the time $\left|T_{0}\right|$ after the acceleration has taken place. For large $\left|T_{0}\right|$ this term is approximately $-E_{r}\left|T_{0}\right| / p$, where $E_{r}$ is the classical energy of the radiation. If the width $\Delta z$ of the wave packet is narrowest with $\Delta z \sim \hbar / \Delta p$ when the particle is going through acceleration [22], then after time $\left|T_{0}\right|$ its square behaves like $(\Delta z)^{2} \sim(\hbar / \Delta p)^{2}+(\Delta p / m)^{2}\left|T_{0}\right|^{2}$. The second term dominates for large enough $\left|T_{0}\right|$. Therefore, in theory, one can make the position shift larger than the width of the wave packet by making $\left|T_{0}\right|$ large and letting $\Delta p$ satisfy

$$
\Delta p<\frac{m E_{r}}{p} \sim \frac{\alpha \hbar m a^{2} t_{a}}{p c^{2}}
$$

where $a$ is the typical acceleration and where $t_{a}$ is the duration of the acceleration 23. Then, with $p=m v$,

$$
\Delta z \sim \frac{\hbar}{\Delta p} \gtrsim \frac{1}{\alpha}\left(\frac{c}{a t_{a}}\right)^{2} z_{a} \gg z_{a},
$$

where $z_{a}=v t_{a}$ is the length of the interval in $z$ where the acceleration occurs. Thus, the wave packet would be much wider than the region of acceleration if the position shift were to be larger, in theory, than the width of the wave packet at a later time.

\section{ERRATUM}

In finding the $\hbar \rightarrow 0$ limit of the scattering amplitude [Eq. (59)] we erroneously discarded surface terms. We correct this error here. This correction changes our conclusion completely: the position shift in the LorentzDirac theory agrees with that in quantum field theory in the $\hbar \rightarrow 0$ limit.

Since the function $K(z)$ in Eq. (43) can be approximated in the limit $\hbar \rightarrow 0$ by $\omega / v_{p}(z)$ [see Eq. (55)] we have

$$
\begin{aligned}
G\left(k_{z}, P, p\right) & =2 p \int_{-\infty}^{+\infty} d z \exp \left(i \int_{0}^{z} \omega / v_{p}\left(z^{\prime}\right) d z^{\prime}\right) \\
& =2 p \int_{-\infty}^{+\infty} v_{p}(t) e^{i \omega t}
\end{aligned}
$$

(Note that $P \rightarrow p$ in the $\hbar \rightarrow 0$ limit.) Since this integral is ill-defined, we insert a damping factor $\chi(t)$ which takes the value 1 while $a_{p}(t)=v_{p}^{\prime}(t) \neq 0$ and goes to zero smoothly as $t \rightarrow \pm \infty$. Thus,

$$
G\left(k_{z}, P, p\right)=2 p \int_{-\infty}^{+\infty} v_{p}(t) \chi(t) e^{i \omega t} .
$$

The quantity $\hat{a}_{p}(\omega)$ in Eq. (89) needs to be replaced by

$$
-i \omega \int_{-\infty}^{+\infty} v_{p}(t) \chi(t) e^{i \omega t}=\hat{a}_{p}(\omega)+\int_{-\infty}^{+\infty} v_{p}(t) \chi^{\prime}(t) e^{i \omega t} .
$$

Then the contribution of radiation reaction to the position shift is

$$
\begin{aligned}
\langle z\rangle_{t=0}^{\mathrm{rad}}= & \frac{e^{2}}{6 \pi c^{3}} \int_{-\infty}^{+\infty} \frac{d \omega}{2 \pi} \\
& \times\left[\hat{a}_{p}(-\omega)+\int_{-\infty}^{+\infty} v_{p}\left(t^{\prime}\right) \chi^{\prime}\left(t^{\prime}\right) e^{-i \omega t^{\prime}} d t^{\prime}\right] \\
& \times \int_{-\infty}^{+\infty} d t \frac{\partial v_{p}(t)}{\partial p} \chi(t) e^{i \omega t} \\
= & \frac{e^{2}}{6 \pi c^{3}} \int_{-\infty}^{+\infty} d t \\
& \times\left\{a_{p}(t) \frac{\partial v_{p}(t)}{\partial p}+\frac{1}{2} v_{p}(t) \frac{\partial v_{p}(t)}{\partial p} \frac{d}{d t}[\chi(t)]^{2}\right\} .
\end{aligned}
$$


The quantity $\partial v_{p}(t) / \partial p$ can be found as follows. Note that

$$
t=\int_{z_{0}}^{z} \frac{m}{\sqrt{p^{2}-2 m V(\xi)}} d \xi
$$

and hence

$$
d t=\frac{1}{v_{p}(t)} d z_{p}-\frac{v_{f}}{m}\left(\int_{0}^{t} \frac{d \tau}{\left[v_{p}(\tau)\right]^{2}}\right) d p .
$$

This can be re-arranged as

$$
d z_{p}=v_{p}(t) d t+\frac{v_{f}}{m} v_{p}(t)\left(\int_{0}^{t} \frac{d \tau}{\left[v_{p}(\tau)\right]^{2}}\right) d p
$$

By using equality of mixed partial derivatives,

$$
\frac{\partial}{\partial p}\left(\frac{\partial z_{p}}{\partial t}\right)=\frac{\partial}{\partial t}\left(\frac{\partial z_{p}}{\partial p}\right)
$$

we obtain

$$
\frac{\partial}{\partial p} v_{p}(t)=\frac{v_{f}}{m}\left[\frac{1}{v_{p}(t)}+a_{p}(t) \int_{0}^{t} \frac{d \tau}{\left[v_{p}(\tau)\right]^{2}}\right]
$$

By substituting this in Eq. (106) we find

$$
\begin{aligned}
\langle z\rangle_{t=0}^{\mathrm{rad}}= & \frac{e^{2} v_{f}}{6 \pi m c^{3}} \int_{-\infty}^{+\infty} d t \\
& \times\left\{\frac{a_{p}(t)}{v_{p}(t)}+\left[a_{p}(t)\right]^{2} \int_{0}^{t} \frac{d \tau}{\left[v_{p}(\tau)\right]^{2}}\right. \\
& \left.-\frac{1}{2} \frac{d}{d t}\left[a_{p}(t) \int_{0}^{t} \frac{d \tau}{\left[v_{p}(\tau)\right]^{2}}\right]\right\} \\
= & \frac{e^{2} v_{f}}{6 \pi m c^{3}}\left\{\log \frac{v_{f}}{v_{i}}\right. \\
& \left.-\int_{-\infty}^{0} \frac{d \tau}{\left[v_{p}(\tau)\right]^{2}}\left(\int_{-\infty}^{\tau}\left[a_{p}(t)\right]^{2} d t\right)\right\}
\end{aligned}
$$

where we have used the assumption that $a_{p}(t)=0$ if $t \geq$ 0 . This is exactly the formula obtained by integrating Eq. (12) found using the Lorentz-Dirac theory from $t=-\infty$ to $t=0$. Notice that we did not use the assumption that $2 m|V(z)| \ll p^{2}$.

\section{Acknowledgments}

The author thanks Bernard Julia and Mitch Pfenning for useful discussions and the anonymous referee for helpful comments.

\section{APPENDIX A: CALCULATIONS WITHOUT CHOOSING PHYSICAL POLARIZATION STATES}

If we do not use physical polarization states for photons and stick to the Fock space with indefinite metric for the Feynman gauge, the one-photon final state is not given by Eq. (70) but by

$$
\begin{aligned}
|f, 1 \gamma\rangle= & i \int \frac{d^{3} \mathbf{k}}{2 \hbar \omega(2 \pi)^{3}} \int \frac{d^{3} \mathbf{P}}{2 P_{0}(2 \pi \hbar)^{3}} \int \frac{d^{3} \mathbf{p}}{\sqrt{2 p_{0}}(2 \pi \hbar)^{3}} \\
& \times f(\mathbf{p}) \mathcal{A}^{\mu}(\mathbf{k} ; \mathbf{P} ; \mathbf{p}) b_{\mu}^{\dagger}(\mathbf{k}) A^{\dagger}(\mathbf{P})|0\rangle,
\end{aligned}
$$

where

$$
\mathcal{A}_{\mu}(\mathbf{k} ; \mathbf{P} ; \mathbf{p})=-i e \hbar c^{2} \int d t d^{3} \mathbf{x} \Phi_{\mathbf{P}}(t, \mathbf{x})^{*} \stackrel{\leftrightarrow}{\partial}_{\mu} \Phi_{\mathbf{p}}(t, \mathbf{x})
$$

One may assume that $\mathbf{P}_{\perp}$ and $\mathbf{p}_{\perp}$ are negligibly small as before. Let us define $G_{\mu}\left(k_{z}, P, p\right)$ by

$$
\begin{aligned}
& \mathcal{A}_{\mu}(\mathbf{k}, \mathbf{P}, \mathbf{p}) \\
& \equiv e c^{2} G_{\mu}\left(k_{z}, P, p\right) \\
& \quad \times(2 \pi \hbar)^{3} \delta^{2}\left(\mathbf{P}_{\perp}+\hbar \mathbf{k}_{\perp}-\mathbf{p}_{\perp}\right) \delta\left(P_{0}+\hbar \omega-p_{0}\right) .
\end{aligned}
$$

It is clear that $G_{x}$ and $G_{y}$ are negligibly small and that $G_{0}$ and $G_{z}$ are independent of the direction of $\mathbf{k}_{\perp}$. We have

$$
G_{z}\left(k_{z}, P, p\right)=G\left(k_{z}, P, p\right),
$$

where $G\left(k_{z}, P, p\right)$ is defined by Eq. (42). The 0component $G_{0}\left(k_{z}, P, p\right)$ is

$$
\begin{aligned}
G_{0}\left(k_{z}, P, p\right)= & -\sqrt{P p} \frac{p_{0}+P_{0}}{c} \int \frac{d z}{\left[\kappa_{P}(z) \kappa_{p}(z)\right]^{1 / 2}} \\
& \times \exp \left[i \int_{0}^{z} K\left(z^{\prime}\right) d z^{\prime}\right],
\end{aligned}
$$

where $K(z)$ is defined by Eq. (44). Using a formula similar to Eq. (45), we arrive at

$$
\begin{aligned}
G_{0}\left(k_{z}, P, p\right)= & -i \sqrt{P p} \frac{p_{0}+P_{0}}{c} \int d z \\
& \times \frac{d}{d z}\left\{\left[\kappa_{P}(z) \kappa_{p}(z)\right]^{1 / 2} K(z)\right\}^{-1} \\
& \times \exp \left[i \int_{0}^{z} K\left(z^{\prime}\right) d z^{\prime}\right]
\end{aligned}
$$

At lowest order in $\hbar$ we can let $P=p, P_{0}=p_{0}, \kappa_{P}(z)=$ $\kappa_{p}(z) \equiv m v_{p}(z)$ and use the approximation (54). Thus, we find

$$
\begin{aligned}
G_{0}\left(k_{z}, P, p\right)= & -\frac{2 i p p_{0}}{c} \int_{-\infty}^{+\infty} d z \frac{k_{z} v_{p}^{\prime}(z)}{m\left[\omega-v_{p}(z) k_{z}\right]^{2}} \\
& \times \exp \left\{i \int_{0}^{z}\left[\omega / v_{p}\left(z^{\prime}\right)-k_{z}\right] d z^{\prime}\right\} .
\end{aligned}
$$


Then, by letting $p_{0}=m c^{2}$ and $k_{z}=0$ on the right-hand side [because $\omega \gg v_{p}(z) k_{z}$ ] and comparing the result with Eq. (56) we find

$$
G_{0}\left(k_{z}, P, p\right)=-\frac{c k_{z}}{\omega} G\left(k_{z}, P, p\right) .
$$

Thus, the only difference between the calculations here and those in Secs. [II] and [IV] is that the factor $\left|\epsilon^{(1) z}(\mathbf{k}) G\left(k_{z}, P, p\right)\right|^{2}$ is replaced here by $-G_{\mu}\left(k_{z}, P, p\right) G^{\mu}\left(k_{z}, P, p\right)=\left(1-k_{z}^{2} c^{2} / \omega^{2}\right)\left|G\left(k_{z}, P, p\right)\right|^{2}$ in the k-integral for the final results. For a given $\omega$ the average of $1-k_{z}^{2} c^{2} / \omega^{2}$ over the solid angle is $2 / 3$. Since the average of $\left|\epsilon^{(1) z}(\mathbf{k})\right|^{2}$ is also $2 / 3$, we arrive at the same transition probability (77) and position shift (93).

\section{APPENDIX B: A DERIVATION OF THE POSITION EXPECTATION VALUE}

In this Appendix we derive Eq. 800 which was used to find the expected value of the $z$-coordinate of the particle. A systematic method to obtain a wave function for a oneparticle state in the scalar field theory has been given by Feshbach and Villars [19, 20]. We will not use this method explicitly, but the derivation we describe here is based on the idea spelled out in Ref. 19].

Recall that the charge density operator is

$$
\rho=\frac{i}{\hbar c^{2}}\left(\psi^{\dagger} \partial_{t} \psi-\psi \partial_{t} \psi^{\dagger}\right) .
$$

Let us define

$$
\rho_{i}(t, \mathbf{x}) \equiv\langle i|\rho(t, \mathbf{x})| i\rangle,
$$

where the state $|i\rangle$ is defined by Eq. (66). Since this state is a one-particle state, the charge density $\rho_{i}(t, \mathbf{x})$ coincides with the probability density function for the position of the particle at each time up to a normalization factor (which will turn out to be one). By a straightforward calculation we find

$$
\rho_{i}(t, \mathbf{x})=i \hbar \phi_{i}(t, \mathbf{x})^{*} \stackrel{\leftrightarrow}{\partial_{t}} \phi_{i}(t, \mathbf{x}),
$$

where

$$
\begin{aligned}
\phi_{i}(t, \mathbf{x}) & \equiv \frac{1}{\hbar c}\langle 0|\psi(t, \mathbf{x})| i\rangle \\
& =\int \frac{d^{3} \mathbf{p}}{\sqrt{2 p_{0}}(2 \pi \hbar)^{3}} f(\mathbf{p}) \Phi_{\mathbf{p}}(t, \mathbf{x}) .
\end{aligned}
$$

Then it follows from orthonormality (32) of $\Phi_{\mathbf{p}}(t, \mathbf{x})$ and the normalization condition (67) for $f(\mathbf{p})$ that

$$
\int d^{3} \mathbf{x} \rho_{i}(t, \mathbf{x})=1
$$

Thus, the function $\rho_{i}(t, \mathbf{x})$ is the normalized probability density function for the position of the particle. By substituting Eq. (B4) in Eq. (B3) we obtain

$$
\begin{aligned}
\rho_{i}(t, \mathbf{x})=\frac{1}{2} \int \frac{d^{3} \mathbf{p}}{(2 \pi \hbar)^{3}} \int \frac{d^{3} \mathbf{p}^{\prime}}{(2 \pi \hbar)^{3}}\left(\sqrt{\frac{p_{0}^{\prime}}{p_{0}}}+\sqrt{\frac{p_{0}}{p_{0}^{\prime}}}\right) \\
\\
\times f\left(\mathbf{p}^{\prime}\right)^{*} f(\mathbf{p}) \Phi_{\mathbf{p}^{\prime}}(t, \mathbf{x})^{*} \Phi_{\mathbf{p}}(t, \mathbf{x}),
\end{aligned}
$$

where $p_{0}$ is defined by Eq. (26) with $p_{0}^{\prime}$ similarly defined.

The expected value of the $z$-coordinate at $t=0$ is

$$
\langle z\rangle_{t=0}^{\text {off }}=\int d^{3} \mathbf{x} z \rho_{i}(0, \mathbf{x}) .
$$

By using the assumption that the wave packet is located far into the region where $V(z)=0$ at $t=0$ we may approximate the mode function $\Phi_{\mathbf{p}}(0, \mathbf{x})$ at $t=0$ in Eq. (B6) as

$$
\Phi_{\mathbf{p}}(0, \mathbf{x}) \approx \exp \left[\frac{i}{\hbar}\left(p z+\mathbf{p}_{\perp} \cdot \mathbf{x}_{\perp}\right)\right]
$$

With this approximation we can write

$$
z \Phi_{\mathbf{p}}(0, \mathbf{x}) \approx-i \hbar \frac{\partial}{\partial p} \Phi_{\mathbf{p}}(0, \mathbf{x}) .
$$

By substituting this formula in Eq. (B7) and integrating by parts we find

$$
\begin{aligned}
\langle z\rangle_{t=0}^{\mathrm{off}}= & \frac{i \hbar}{2} \int \frac{d^{3} \mathbf{p}}{(2 \pi \hbar)^{3}} \int \frac{d^{3} \mathbf{p}^{\prime}}{(2 \pi \hbar)^{3}} \frac{\partial}{\partial p}\left[\left(\sqrt{\frac{p_{0}}{p_{0}^{\prime}}}+\sqrt{\frac{p_{0}^{\prime}}{p_{0}}}\right) f\left(\mathbf{p}^{\prime}\right)^{*} f(\mathbf{p})\right] \int d^{3} \mathbf{x} \Phi_{\mathbf{p}^{\prime}}(0, \mathbf{x})^{*} \Phi_{\mathbf{p}}(0, \mathbf{x}) \\
= & i \hbar \int \frac{d^{3} \mathbf{p}}{(2 \pi \hbar)^{3}} \int \frac{d^{3} \mathbf{p}^{\prime}}{(2 \pi \hbar)^{3}} \\
& \times\left[\frac{c^{2}}{2}\left(\frac{1}{\sqrt{p_{0}^{\prime} p_{0}}}-\sqrt{\frac{p_{0}^{\prime}}{p_{0}^{3}}}\right) f\left(\mathbf{p}^{\prime}\right)^{*} f(\mathbf{p})+f\left(\mathbf{p}^{\prime}\right)^{*} \frac{\partial}{\partial p} f(\mathbf{p})\right](2 \pi \hbar)^{3} \delta^{3}\left(\mathbf{p}-\mathbf{p}^{\prime}\right) \\
= & i \hbar \int \frac{d^{3} \mathbf{p}}{(2 \pi \hbar)^{3}} f(\mathbf{p})^{*} \frac{\partial}{\partial p} f(\mathbf{p}) .
\end{aligned}
$$

We immediately obtain Eq. (80) from this formula by integration by parts. 
[1] H. A. Lorentz, Theory of electrons, (Dover, New York, 1952).

[2] P. A. M. Dirac, Classical theory of radiating electrons, Proc. Roy. Soc. London A167, 148 (1938).

[3] J. D. Jackson, Classical electrodynamics, (Wiley, New York, 1975)

[4] F. Rohrlich, Classical charged particles, (Addison-Wesley, Redwood City, 1990).

[5] G. N. Plass, Classical electrodynamic equations of motion with radiative reaction, Rev. Mod. Phys. 33, 37 (1961).

[6] F. Rohrlich, The equations of motion of classical charges, Ann. Phys. (N.Y.) 13, 93 (1961).

[7] A. D. Yaghjian, Relativistic dynamics of a charged sphere, (Springer, Berlin, 1992).

[8] L. D. Landau and E. M. Lifshitz, The classical theory of fields, (Pergammon, Oxford, 1962).

[9] E. E. Flanagan and R. M. Wald, Do back reaction enforce the averaged null energy condition in semiclassical gravity? Phys. Rev. D 54, 6233 (1996).

[10] E. Poisson, An introduction to the Lorentz-Dirac equation, ArXiv:gr-qc/9912045

[11] E. J. Moniz and D. H. Sharp, Absence of runaways and divergent self-mass in nonrelativistic quantum electrodynamics, Phys. Rev. D 10, 1133, (1974); ibid. Radiation reaction in nonrelativistic quantum electrodynamics, 15, 2850, (1977).

[12] Y. Nambu, $S$ matrix in semiclassical approximation, Phys. Lett. 26B, 626 (1968).

[13] L. S. Brown and D. Boulware, Tree graphs and classical fields, Phys. Rev. 172, 1628 (1968).

[14] C. Itzykson and J.-B. Zuber, Quantum field theory, (McGraw-Hill, New York, 1980).
[15] B. L. Julia, Dualities in the classical supergravity limits: Dualisations, dualities and a detour via $4 k+2 d i-$ mensions, in Cargèse 1997, Strings, branes and dualities, 121-139, LPTENS-98-07, arXiv:hep-th/9805083

[16] A. Higuchi, Radiation reaction in quantum mechanics, arXiv:quant-ph/9812036 unpublished.

[17] M. Abraham, Theorie der Elektrizität, Vol. II, (Springer, Leipzig, 1905).

[18] L. I. Schiff, Quantum mechanics, (McGraw-Hill, New York, 1968).

[19] H. Feshbach and F. Villars, Elementary relativistic wave mechanics of spin 0 and spin 1/2 particles, Rev. Mod. Phys. 30, 24 (1958).

[20] A. S. Davydov, Quantum Mechanics, (Pergamon, Oxford, 1965).

[21] The corresponding correction to the forward-scattering amplitude is of order $\hbar^{-2}$. Since the imaginary part of $\mathcal{F}$ is of order $\hbar^{-1}$, the correction to the potential does not have an imaginary part at leading order in $\hbar$.

[22] The wave packet (68) is narrowest at $t=0$ when the position is measured. One can make this wave packet more general so that the case considered here, which is more realistic, is included by changing the factor $e^{-i p z_{0} / \hbar}$ to $e^{-i h(p) / \hbar}$ with $h^{\prime}(\bar{p})=z_{0}$. The position shift will be unchanged with this modification.

[23] Since the second term in Eq. (17) is the lowest-order term in $V(z)$ that grows linearly in $\left|T_{0}\right|$, the fact that it is much larger than the first term, which is of lower order in $V(z)$, does not imply that our approximation breaks down since the latter is independent of $\left|T_{0}\right|$. 